\title{
Effect of Grain Misorientation and Martensitic Transformation on Surface Roughening Behavior in Thin Austenitic Stainless Steel Foils
}

\author{
Abdul Aziz ${ }^{1 *}$, Ming Yang1 ${ }^{1}$, Tetsuhide Shimizu1, Tsuyoshi Furushima² \\ ${ }^{1}$ Tokyo Metropolitan University, Hino Campus, 6-6-Asahigaoka, Hinoshi, Tokyo 191-0065, Japan \\ ${ }^{2}$ Institute of Industrial Science, The University of Tokyo, 4-6-1, Meguro City, Tokyo 153-8505, Japan
}

\begin{abstract}
Stainless steel thin foils have unlimited applications in the field of microforming industries, making them attractive for use in industrial society. The problem hindering their use is the different mechanical properties that exist between thick and ultra-thin metals with the same plastic deformation characteristics. In this study, we compared the martensitic phase transformation (MPT) effect in SUS 304 and SUS 316, which was clarified in samples of the same grain size (Dg). The correlation between the MPT, grain misorientation (GM), and surface roughening behavior of SUS 304 and 316 thin metal foils was investigated using a uniaxial tensile test, which was repeated five times, with constant strain increments of $1.5 \%$. Phase transformations such as MPT and GM were investigated using scanning electron microscopy/electron backscattered diffraction (SEM-EBSD). The results show that surface roughening increased proportionally in both the SUS 304 and SUS 316 thin foils with a coarse grain size (Dg of $9.0 \mu \mathrm{m}$ ). Surface roughening increased to a greater extent in the coarse-grained SUS 304 and 316 thin metal foils compared with the fine-grained (Dg $1.5 \mu \mathrm{m})$ samples. The SEM-EBSD results show that the grain strength of the coarse-grained SUS 316 thin metal foil was less inhomogeneous than that of the coarse-grained SUS 304 thin metal foil. The surface roughness ratio of the coarse-grained SUS 304 was higher than that of the coarse-grained SUS 316. The inhomogeneous grain strength of the fine-grained SUS 304 was similar to that of the fine-grained SUS 316. The surface roughness ratio of the fine-grained SUS 304 was similar to that of the fine-grained SUS 316. The MPT demonstrated a huge effect on the surface roughening behavior of the SUS 304 and SUS 316 samples with different Dg.
\end{abstract}

Keywords: Grain Size (Dg); Grain Misorientation (GM); Martensitic Phase Transformation (MPT)

\section{Introduction}

Austenitic stainless steel foils have a wide range of applications in many industries, such as the electric power, electronics, biomedicals, nuclear, and food. Because of the high demand for microparts, austenitic stainless steel foils have received a great deal of attention (Aziz and Yang, 2020). The uniaxial tensile test induces a martensitic phase transformation (MPT) in stainless steel. The martensite phase volume fraction (Mf) increases proportionally after plastic deformation is applied to austenitic stainless steel foils (Engel and Eckstein, 2002; Xue et al., 2010). The effects of MPT on stainless steel include an increase in strength and a decrease in toughness (Milad et al., 2008; Jha et al., 2008). In previous studies, the Mf occurred in a strip of stainless steel after plastic deformation

*Corresponding author's email: aziz-abdul@ed.tmu.ac.jp, Tel.: +81-07042850529 doi: 10.14716/ijtech.v12i6.5180 
(Xue et al., 2010; Qin and Xia, 2020; Suryadi et al., 2020). MPT nucleation originates from the shear band intersection in stainless steel after plastic deformation (Tomita and Iwamoto,1995). Until recently, investigations focusing on surface roughness behavior in thin metal foils with an FCC structure have been rare, highlighting the need for further study in this area (Fauzun et al., 2011; Zhang et al., 2017; Dewi et al., 2020). The most important factor affecting formability in thin or sheet metal is surface roughening and not voids (Cheng et al., 2017). Surface roughness depends on the grain size (Dg), and an increase in the Dg leads to a decrease in the ratio of thickness to Dg; thus, there is a need to investigate the surface roughness behavior of thin metal foils with a Dg of less than $10 \mu \mathrm{m}$ (Yoshida, 2014). The deformation of different individual grains affects the surface roughening behavior of sheet metal (Ichiro et al., 2001; Citrawati et al., 2020). In a weak grain, deformation affects the surface roughening behavior of thin metal foils and sheet metal (Furushima et al., 2013). Without annealing, coarse grain $(\mathrm{Dg}=9.0 \mu \mathrm{m})$ deformation causes a proportional increase in surface roughness during uniaxial tests, whereas in fine grain (Dg 3.0 and $1.5 \mu \mathrm{m}$ ) deformation, the surface roughness increase is not proportional. The MPT has been shown to affect the surface roughening behavior of coarse-grained SUS 304 (Aziz and Yang, 2020). The ductility of SUS 304 and SUS 316 could be enhanced via annealing treatment (Shuro et al., 2010).

Based on the findings above, the effect of MPT on the surface roughening behavior of SUS 304 and SUS 316 thin metal foils with different and lower grain sizes still needs to be clarified. Until recently, a limited amount of research has investigated the effect of MPT and grain misorientation (GM) on surface roughening behavior and its associated mechanism.

The objectives of this research were to investigate the effects of MPT and GM on the surface roughening behavior of SUS 304 and SUS 316 thin foils with different grain sizes (Dg) of $1.5 \mu \mathrm{m}$ and $9.0 \mu \mathrm{m}$. To achieve these objectives, thin metal foils were subjected to a uniaxial tensile test. The uniaxial tensile test was carried out five times, and the surface roughness behavior was measured at every step. After performing the uniaxial tensile test five times, SEM-EBSD was used to analyze the phase mapping and the GM behavior.

\section{Methods}

After the rolling treatment, the thin austenitic stainless steel metal (SUS 304 and SUS 316) samples had a thickness of $0.1 \mathrm{~mm}$ and grain sizes (Dg) of $1.5 \mu \mathrm{m}$ and $9.0 \mu \mathrm{m}$. SUS 304 and SUS 316 thin metal foils were chosen for use as specimens because SUS 304 has a more complicated microstructure than SUS 316, which allowed them to be compared to each other more easily. The microstructures of SUS 304 and SUS 316 influence the formation of the MPT and the surface roughening behavior. In this research, the authors aimed to clarify the surface roughening mechanism in SUS 304 and SUS 316 thin metal foils with a Dg of less than $10 \mu \mathrm{m}$. SUS 304 and SUS 316 thin metal foils were supplied by Komatsu Seiki Koshakuso Co. Ltd., Suwa City, Nagano, Japan. To investigate the effects of MPT and GM on the surface roughening behavior of SUS 304 and SUS 316 thin metal foils with different grain sizes, the materials were heat-treated in annealing conditions at $400^{\circ} \mathrm{C}$ for $1 \mathrm{~h}$ to relax the samples.

Before the thin metal foils were subjected to the uniaxial tensile stress state (with increments of $1.5 \%$ strain added during each step), the surfaces of the samples were cleaned. To clean the surface of the metal, the thin metal foils were washed using ethanol combined with ultrasonic vibration for $30 \mathrm{~min}$.

The uniaxial tensile test was performed using a Shimadzu Tensile Machine (Shimadzu Corp., Japan) with a $5 \mathrm{kN}$ capacity. The AGX-50KNVD model of the Shimadzu Tensile Machine was used for the uniaxial tensile test, which was applied to the specimens in five stages. After the samples were in the uniaxial tensile stress state, surface roughness was 
measured. The uniaxial tensile stress state and surface roughness measurements were repeated five times. Surface roughness was measured using a confocal laser microscope (OLS-5000; Olympus, Corp., Japan). SEM-EBSD was used to analyze the microstructure of the foils after the completion of the tensile tests through phase mapping and grain misorientation analysis. The microstructure behavior of each specimen was investigated using SEM SU-70 (Hitachi High Technology, Japan). The SEM SU-70 specifications were an emission current of $16 \mu \mathrm{A}$, an acceleration voltage of $5 \mathrm{kV}$, and a working distance of $10 \mathrm{~mm}$. The EBSD Digi View (EDAX) in field-free mode was used to investigate the phase transformations. The EBSD settings were an emission current of $16 \mu \mathrm{A}$, a working distance of $20 \mathrm{~mm}$, and an acceleration voltage of $15 \mathrm{kV}$. The EBSD sample observation area was 30 $\mu \mathrm{m} \times 50 \mu \mathrm{m}$, the EBSD pixel binning area was $8 \times 8$, and the resolution of the EBSD machine was $0.1 \mu \mathrm{m}$.

\section{Results and Discussion}

Previous investigations determining the surface roughening mechanism in fine-grained SUS 304 and SUS 316 and coarse-grained SUS 304 without annealing determined that the MPT has an effect on the surface roughening behavior in coarse-grained SUS 304 (Aziz and Yang, 2020). In this research, the authors investigated the effects of MPT and GM on both fine-grained and coarse-grained SUS 304 and SUS 316 thin foils with the same grain size (Dg) after annealing treatment. The stress-strain curves that were obtained from the tensile test are shown in Figure 1. The tensile strength and ductility differed between specimens with the same grain size; the tensile strength and ductility of the SUS 304 thin foil were higher than those of SUS 316. For both materials, the tensile strength of the finegrained sample was higher than that of the coarse-grained sample. Figures 2 and 3 show the surface roughening behavior of both materials with different grain sizes (Dg). With the same strain, the surface roughening $(\mathrm{Ra})$ behavior increased proportionally for the coarsegrained SUS 316 and SUS 304 thin metal foils. The Ra ratio of SUS 304 was higher than that of SUS 316, as shown in Figure 2. Furthermore, a comparison of Figures 2 and 3 reveals that the Ra ratio was greater for the coarse-grained foils than for the fine-grained foils for both materials.

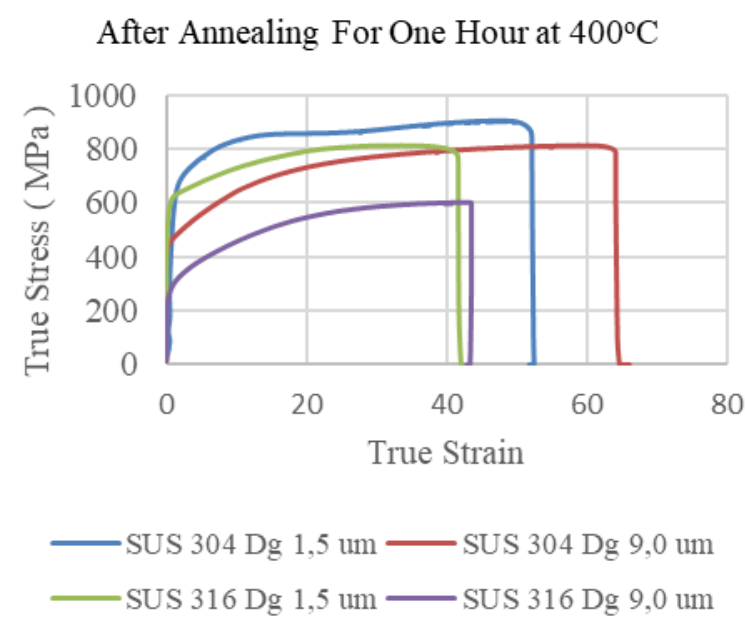

Figure 1 Material deformation behavior

Figures 4 and 5 show the SEM-EBSD results that were used for the MPT and GM analysis. The MPT and austenite phases are shown in the phase maps in Figure 4. The red area is austenite, and the green area is the MPT. 


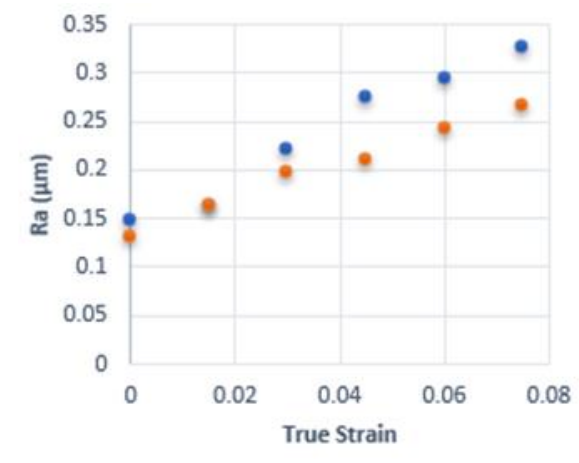

- Dg 9,0 um SuS $304 \quad$ Dg 9,0 um SUS 316

Figure 2 Surface roughening in Dg $9.0 \mu \mathrm{m}$

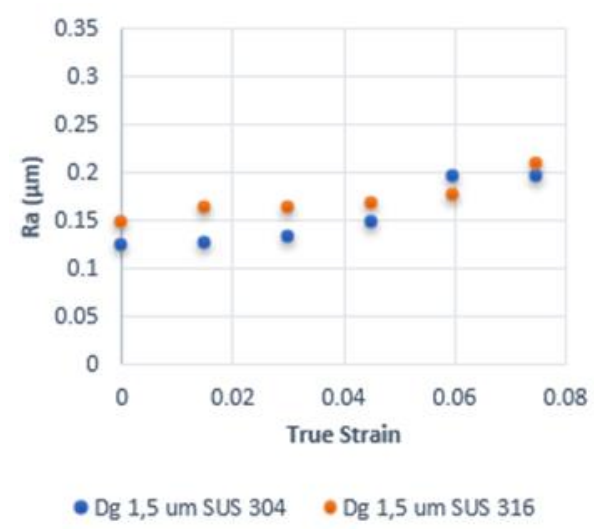

Figure 3 Surface roughening in Dg 1.5 um

In Figure 5, the green, red, and blue areas are the results of kernel average misorientation analysis (KAM) mapping. Blue indicates a misorientation of $0^{\circ}$, and green and red indicate a misorientation of approximately $2^{\circ}$ and $5^{\circ}$, respectively. The misorientation calculation is based on the average misorientation in the center and the surrounding misorientation in the kernel is the KAM map (12). The MPT and GM occurred in SUS 304 with a different Dg, but the MPT did not occur in the fine- or coarse-grained SUS 316, as shown in Figures 4 and 5. The GM occurred in the SUS 316 samples with fine and coarse Dg, as shown in Figures 4 and 5 .

As shown in Figures 2 and 3, the coarse- and fine-grained foils differed in terms of their surface roughening behavior. The surface roughening behavior of both the coarse- and finegrained foils increased proportionally. The surface roughening behavior of the coarsegrained foils was higher than that of the fine-grained samples. The coarse-grained specimens had higher ductility than fine-grained foils, as shown in Figure 1. Higher ductility was associated with an increase in the surface roughness value (Aziz and Yang, 2020).

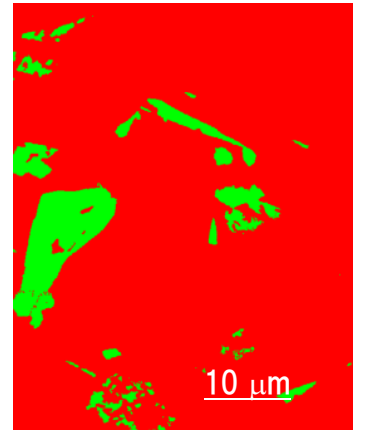

SUS $304 \mathrm{Dg} 9.0 \mu \mathrm{m}$

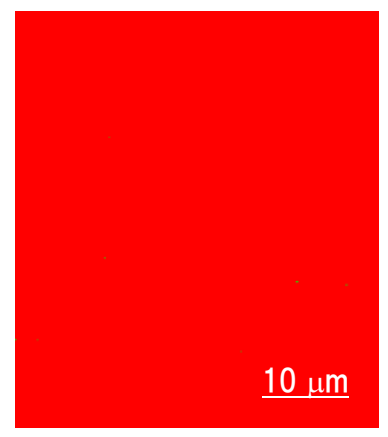

SUS 316 Dg $9.0 \mu \mathrm{m}$

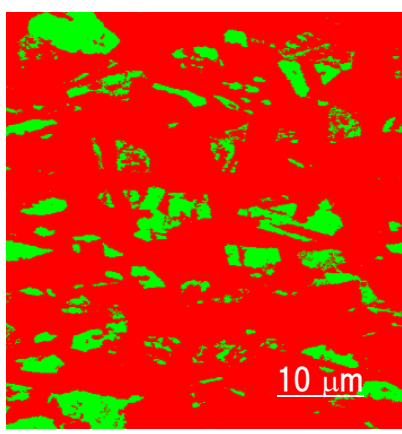

SUS $304 \mathrm{Dg} 1.5 \mu \mathrm{m}$

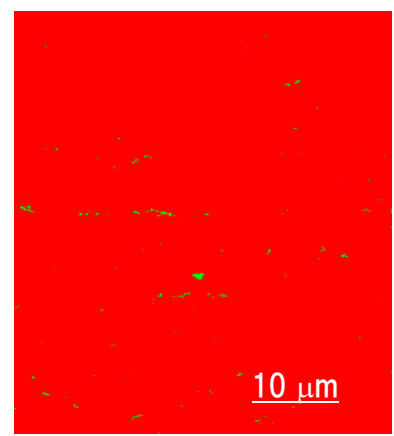

SUS 316 Dg $1.5 \mu \mathrm{m}$

$=$ MPT

= Austenite Phase

Figure 4 EBSD phase mapping at 7.5\% stress accumulation

After the uniaxial tensile test, the MPT was more extensive, larger, and more uniform in the fine-grained SUS 304 thin foil than in its coarse-grained counterpart. The MPT was extensive in the fine-grained SUS 304 thin foil after reaching the uniaxial tensile stress state, affecting the homogeneous grain strength. The MPT did not occur in either the fine- or 
coarse-grained SUS 316 thin metal foils. The MPT was not uniform in the coarse-grained SUS 304, which resulted in inhomogeneous grain strength, as shown in Figure 4.

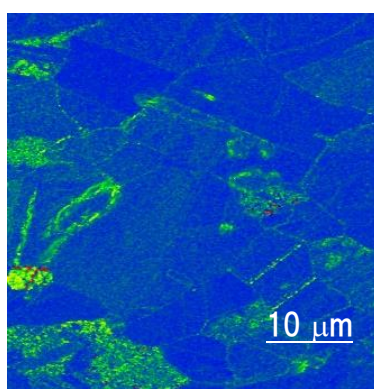

GMSUS 304 Dg $9.0 \mu \mathrm{m}$

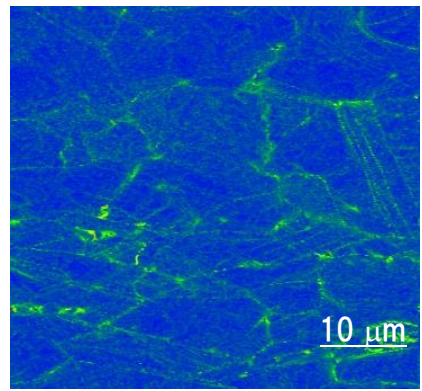

GM SUS 316 Dg $9.0 \mu \mathrm{m}$

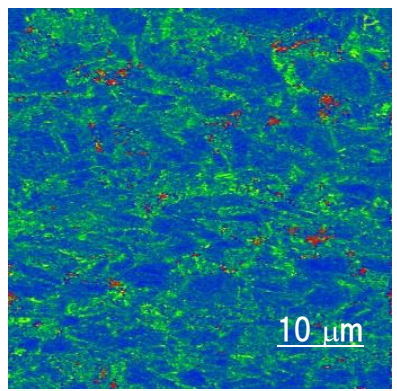

GM SUS 304 Dg $1.5 \mu \mathrm{m}$ $=2^{\circ}$ Misorientation

$=5^{\circ}$ Misorientation

- No Misorientation

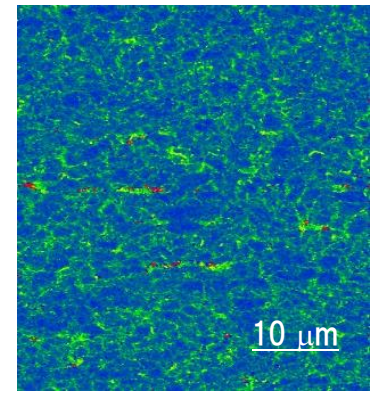

GM SUS 316 Dg $1.5 \mu \mathrm{m}$

Figure 5 EBSD KAM mapping at 7.5\% stress accumulation

The MPT and GM in SUS 316 were less extensive than those in the SUS 304 thin metal foil. This is because the grain strength of SUS 304 thin foil is higher than that of SUS 316, and higher grain strength resists grain deformation. The non-uniform grain strength of the SUS 304 thin foil was associated with more grain strength inhomogeneity. The greater inhomogeneity in the SUS 304 grain strength could give rise to the higher surface roughness of SUS 304 thin foil compared with that of the coarse-grained SUS 316. The coarse-grained SUS 316 and SUS 304 had similarly inhomogeneous grain strength, but the surface roughness of SUS 304 was greater than that of SUS 316, so the effect of the MPT was greater than that of the GM. The fine-grained SUS 304 and SUS 316 had similar surface roughening behaviors because the GM was distributed uniformly in both materials. Uniform MPT occurred in SUS 304. The phase map is shown in Figure 4, and the GM map is shown in Figure 5.

Furthermore, the fine-grained SUS 316 and SUS 304 had a similar GM, which affected the grain strength of the thin foils. The GM was distributed uniformly in the SUS 316 and 304 thin foils. The grain strength of SUS 316 was only affected by the GM, but the grain strength of SUS 304 was affected by both the MPT and the GM. As a result, the grain strength of SUS 304 was higher than that of SUS 316. SUS 316 had a lower grain strength than the SUS 304 thin foil because the grain strength of SUS 316 was only affected by the GM (Aziz and Yang, 2020). SUS 304 had higher ductility than SUS 316 because after annealing at $400^{\circ} \mathrm{C}$, the slip band became more active in SUS 304 than it did in SUS 316 (Tomita and Iwamoto, 1995; Qin and Xia, 2020). In addition, because of the heating process, the $\alpha^{\prime}$ was higher in the SUS 316 thin foils than it was in the SUS 304 thin foils (Shuro et al., 2010). In the fine-grained samples, the grain strength of SUS 304 was higher than that of SUS 316, but the surface roughness was similar because each grain rotated the same angle and in the normal direction during deformation (Ichiro et al., 2001).

The findings obtained in this research show that the MPT and GM produce different effects on the Ra for different Dg. Deformation in a material with an inhomogeneous grain strength affects the Ra, and the values of the GM, MPT, and Dg contribute to inhomogeneous grain strength after deformation. It is important to study MPT, GM, and Dg to clarify the Ra mechanism that occurs during tensile tests in SUS 304 and SUS 316 thin foils. The coarse grain size leads to a higher Ra compared to the fine grain size because the grain strength of the coarse-grained material is more inhomogeneous than that of the fine-grained material. 


\section{Conclusions}

This research investigated the effect of the MPT and GM on the surface roughening behavior of SUS 304 and SUS 316 with the same grain size (Dg) after annealing treatment. The following conclusions can be drawn from the five-stage tensile tests and the SEM-EBSD investigation:

The effect of the MPT on the Ra was greater than that of the GM in the coarse-grained samples. However, the increase in the Ra was greater for the coarse-grained SUS 304 than it was for the fine-grained SUS 304 because the slip band intersection was lower in the coarse-grained sample than it was in the fine-grained specimen, resulting in a lower MPT in the coarse-grained material.

The inhomogeneous grain strength of the coarse-grained SUS 304 was higher than that of the whole material. The Ra of the coarse-grained SUS 304 was higher than that of both the coarse- and fine-grained SUS 316 thin foil.

The grain strength of the coarse-grained SUS 316 was more inhomogeneous than that of the fine-grained SUS 316, resulting in a greater increase in the Ra in the coarse-grained sample. The inhomogeneity of the higher grain strength of the coarse-grained SUS 316 thin foil was affected by its lower GM compared with the fine-grained SUS 316.

The fine-grained SUS 304 and SUS 316 had similar Ra behaviors because of their similar grain strength homogeneity.

\section{Acknowledgements}

The authors would like to express their sincere gratitude and thanks to Komatsuseiki Kosakusho Co. Ltd., especially Tomoaki Yoshino San and Yohei Suzuki San, for providing the samples. The authors are also grateful to Assistant Professor Oshima; Sota, Tokyo Metropolitan University, for lending the Shimadzu Tensile Machine to them.

\section{References}

Aziz, A., Yang, M., 2020. Effect of Martensitic Transformation and Grain Size on Surface Roughening Behavior in SUS 304 and SUS 316 Thin Metal Foils. Eng, Volume 1(2), pp. 167-182

Cheng, C., Wan, M., Meng, B., 2017. Size Effect on the Forming Limit of Sheet Metal in MicroScaled Plastic Deformation Considering Free Surface Roughening. Procedia Engineering, Volume 207, pp. 1010-1015

Citrawati, F., Dwiwandono, R., Firmansyah, L., 2020. The Effect of Ni on the Formation of Bainite in Fe-Ni Laterite Steels Through Semi-Continuous Cooling Method. International Journal of Technology, Volume 11(1), pp. 60-70

Dewi, A.H., Yulianto, D.K., Ana, I.D., Rochmadi., Siswomiharjo, W., 2020. Effect of Cinnamaldehyde, an Anti-Inflammatory Agent, on the Surface Characteristics of a Plaster of Paris - $\mathrm{CaCO}_{3}$ Hydrogel for Bone Substitution in Biomedicine. International Journal of Technology, Volume 11(5), pp. 963-973

Engel, U., Eckstein, R., 2002. Microforming - From Basic Research to Realization. Journal of Materials Processing Technology, Volume 125-126, pp. 35-44

Fauzun., Hamdi, M., Ariga, T., 2011. Effect of the Base Metal Surface Roughness on the Bag8 Spreading Behavior. International Journal of Technology, Volume 2(3), pp. 242-247

Furushima, T., Tsunezaki, H., Nakayama, T., Manabe, K., Alexandrov, S., 2013. Prediction of Surface Roughening and Necking Behavior for Metal Foils by Inhomogeneous FE Material Modelling. Key Engineering Materials, Volume 554-557, pp. 169-173 
Ichiro, S., Takao, O., Takeji, A., Hideaki, T., 2001. Surface Roughening and Deformation of Grains during Uniaxial Tension of Polycrystalline Iron. JSME International Journal Series A, Volume 44, pp. 499-506

Jha, A.K., Sivakumar, D., Sreekumar, K., Mittal, M.C., 2008. Role of Transformed Martensite in the Cracking of Stainless Steel Plumbing Lines. Engineering Failure Analysis, Volume 15(8), pp. 1042-1051

Milad, M., Zreiba, N., Elhalouani, F., Baradai, C., 2008. The Effect of Cold Work on Structure and Properties of AISI 304 Stainless Steel. Journal of Materials Processing Technology, Volume 203(1-3), pp. 80-85

Qin, Z., Xia, Y., 2020. Role of Strain-Induced Martensitic Phase Transformation in Mechanical Response of 304L Steel at Different Strain-Rates and Temperatures. Journal of Materials Processing Technology, Volume 280, pp. 116-613

Shuro, I., Umemoto, M., Todaka, Y., Yokoyama, S., 2010. Phase Transformation and Annealing Behavior of SUS 304 Austenitic Stainless Steel Deformed by High Pressure Torsion. Materials Science Forum, Volume 654, pp. 334-337

Suryadi, S., Kusuma., A.P., Suhadi, A., Priadi, D., 2017. Effect of Annealing Temperature on Microstructure and Mechanical Properties of Ultra Fine Grained Brass Produced by Equal Channel Angular Pressing. International Journal of Technology, Volume 8(1), pp. $58-65$

Tomita, Y., Iwamoto, T., 1995. Constitutive Modeling of TRIP Steel and its Application to The Improvement of Mechanical Properties. International Journal of Mechanical Sciences, Volume 37(12), pp. 1295-1305

Xue, Z.Y., Zhou, S., Wei, X.C., 2010. Influence of Pre-Transformed Martensite on WorkHardening Behavior of SUS 304 Metastable Austenitic Stainless Steel. Journal of Iron and Steel Research International, Volume 17, pp. 51-55

Yoshida, K., 2014. Effect of Grain Scale Heterogeneity on Surface Roughness and Sheet Metal Necking. International Journal of Mechanical Sciences, Volume 83, pp. 48-56

Zhang, L., Xu, W., Liu, C., Ma., X., Long, J., 2017. Quantitative Analysis of Surface Roughness Evolution in FCC Polycrystalline Metal during Uniaxial Tension. Computational Materials Science, Volume 132, pp. 19-29 